\section{A) Check for updates}

Cite this: Org. Biomol. Chem., 2018, 16, 2997

\title{
A rapid and concise setup for the fast screening of FRET pairs using bioorthogonalized fluorescent dyes†
}

\author{
Réka Petrovics, ${ }^{a}$ Bianka Söveges, ${ }^{a}$ Alexandra Egyed, ${ }^{a}$ Gergely Knorr, ${ }^{a}$ \\ Attila Kormos, (D) a Tímea Imre, ${ }^{b}$ György Török, (D) ${ }^{c}$ András Zeke, ${ }^{d}$ Éva Kocsmár, ${ }^{e}$ \\ Gábor Lotz, ${ }^{e}$ Péter Kele (D) a and Krisztina Németh (D) *a
}

\begin{abstract}
One of the most popular means to follow interactions between bio(macro)molecules is Förster resonance energy transfer (FRET). There is large interest in widening the selection of fluorescent FRET pairs especially in the region of the red/far red range, where minimal autofluorescence is encountered. A set of bioorthogonally applicable fluorescent dyes, synthesized recently in our lab, were paired (Cy3T/Cy5T; Cy1A/Cy3T and Cy1A/CBRD1A) based on their spectral characteristics in order to test their potential in FRET applications. For fast elaboration of the selected pairs we have created a bioorthogonalized platform based on complementary 17-mer DNA oligomers. The cyclooctynylated strands were modified nearly quantitatively with the fluorophores via bioorthogonal chemistry steps, using azide- (Cy1; CBRD1) or tetrazine-modified (Cy3; Cy5) dyes. Reactions were followed by capillary electrophoresis using a method specifically developed for this project. FRET efficiencies of the fluorescent dye pairs were compared both in close proximity ( $5^{\prime}$ and $3^{\prime}$ matched) and at larger distance ( $5^{\prime}$ and $5^{\prime}$ matched). The specificity of FRET signals was further elaborated by denaturation and competition studies. Cy1A/Cy3T and Cy1A/CBRD1A introduced here as novel FRET pairs are highly recommended for FRET applications based on the significant changes in fluorescence intensities of the donor and acceptor peaks. Application of one of the FRET pairs was demonstrated in live cells, transfected with labeled oligos. Furthermore, the concise installation of the dyes allows for efficient fluorescence modification of any selected DNA strands as was demonstrated in the construction of Cy3T labeled oligomers, which were used in the FISH-based detection of Helicobacter pylori.
\end{abstract}

Received 25th January 2018, Accepted 29th March 2018 DOI: $10.1039 / c 80 b 00213 d$ rsc.li/obc

\section{Introduction}

${ }^{a}$ Research Centre for Natural Sciences of Hungarian Academy of Sciences, Institute of Organic Chemistry, "Lendület" Chemical Biology Research Group, H-1117 Budapest, Magyar tudósok krt. 2, Hungary. E-mail: nemeth.krisztina@ttk.mta.hu; Tel: +3613826659

${ }^{b}$ Research Centre for Natural Sciences of Hungarian Academy of Sciences, Instrumentation Center, MS Metabolomics Research Group, H-1117 Budapest, Magyar tudósok krt. 2, Hungary

${ }^{c}$ Research Centre for Natural Sciences of Hungarian Academy of Sciences, Institute of Enzymology, Molecular Cell Biology Research Group, H-1117 Budapest,

Magyar tudósok krt. 2, Hungary

${ }^{d}$ Research Centre for Natural Sciences of Hungarian Academy of Sciences, Institute of Enzymology, Protein Research Group, H-1117 Budapest, Magyar tudósok krt. 2, Hungary

${ }^{e} 2^{\text {nd }}$ Department of Pathology, Semmelweis University, Budapest H-1091 Üllői str. 93, Hungary

$\dagger$ Electronic supplementary information (ESI) available: Spectral overlap of the dyes, details of the capillary electrophoretic follow up, the LC-MS spectra of the labeled oligomers, calculation of the oligomer distances, the temperature controlled denaturation of the DNA oligomers, flow cytometric analysis and NMR spectra of Cy1 dye. See DOI: 10.1039/c8ob00213d
Detection of bio(macro)molecules in biological samples or their interactions are preferably followed by fluorescence. ${ }^{1-3}$ The derivatization of target molecules of interest by means of small fluorescent molecules is less laborious than expressing them with fusion tags (e.g. GFP). ${ }^{4}$ Furthermore, small molecules are less likely to perturb the function and interacting characteristics of the protein of interest (POI). Moreover, the use of fusion proteins as tagging agents is limited to proteins, leaving out other biomolecules that are not directly encoded in the genome. ${ }^{5,6}$ Detection of specific oligonucleotide sequences, whose presence is associated with infections or pathophysiological alterations (e.g. cancer), is mostly carried out by labeled counterstrands as hybridization probes. Such diagnostic methods are rationalized by the fluorescent in situ hybridization technique (FISH) or DNA arrays in DNA chips. ${ }^{7-12}$

An alternative setup employing two (or more) fluorophores is possible through the Förster-type resonance energy transfer 
(FRET) effect. FRET is a non-radiative, long-range energy transfer process between two light responsive molecules via dipoledipole interactions. ${ }^{13-16}$ FRET efficiency depends mainly on the spectral overlap integral (including quantum yields) of the partners. Moreover, it is inversely proportional to the sixth power of the distance between the donor and acceptor molecules and highly affected by the orientations of the transition dipoles of the donor and the acceptor moieties. ${ }^{17,18}$ Since FRET based assays are widely used in research and in clinical diagnostics e.g. in the $5^{\prime}$ nuclease assay for PCR monitoring (TaqMan probes), single nucleotide polymorphism (SNP) detection, real-time nucleic acid detection, multiplex real-time PCR quantification, allelic discrimination, and cleavage and mutation detection ${ }^{7,19-22}$ there is great interest in widening the selection of available fluorescent FRET pairs, especially in the orange/red/far red range. Furthermore, fluorophores with large Stokes shifts are ideal candidates for FRET applications as they prevent direct excitation of acceptor emission by the light used to excite the donor. ${ }^{23}$ Installation of fluorophores onto oligonucleotides is crucial. Bioorthogonal chemical transformations offer fluorescent modulation schemes that are high yielding, biocompatible and proceed even inside cells. Of the bioorthogonal reactions the strain-promoted azide-alkyne cycloaddition (SPAAC) and the inverse electron demand DielsAlder reaction (IEDDA) of tetrazines and strained olefins are the most popular ones due to their full biocompatibility and reasonable reaction rates. ${ }^{23}$ Bioorthogonalization of oligonucleotides can be carried out through post-synthetic modification with biorthogonal handles via reactive functional group modifiers conjugated mostly at the termini of the oligonucleotide strand. ${ }^{5,24}$ Internal incorporation of non-canonical nucleotides harboring a reactive motif is also possible either through the post-synthetic transformation of modified monomers or via the enzymatic incorporation of bioorthogonalized nucleotides. ${ }^{25}$ Accordingly, fluorescent labeling of oligonucleotides is accomplished in a subsequent step using fluorescent probes carrying the complementary biorthogonal function.

Fluorescently labeled double stranded DNAs are ideal models for screening fluorescent dyes for FRET applications. Upon varying the oligomer length the Förster radii can also be estimated. $^{26,27}$

In this study we aimed to test selected fluorescent dye pairs from our dye-set in FRET applications. Selection criteria were based on the Stokes-shifts and excitation/emission bands of the dyes.

\section{Results and discussion}

\section{Selection of dye pairs for efficient FRET}

Firstly, we determined the fluorescence spectra of the dyes synthesized in our lab and paired them according to the spectral overlaps between absorption (acceptors) and emission spectra (donors) (Fig. 1). From the screening procedure the following three pairs seemed to be promising: Cy3-tetrazine/Cy5-tetrazine; Cy1-azide/Cy3-tetrazine; and Cy1-azide/CBRD1-azide
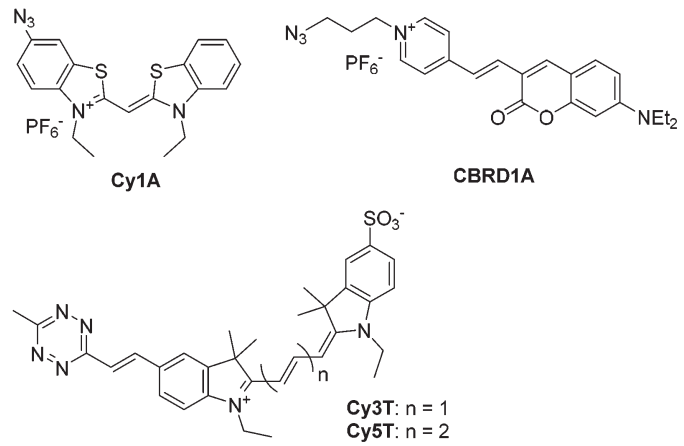

Fig. 1 Structures of the selected dyes for fluorescent labeling of DNA oligomers.

(Chemical Biology Research Group dye 1: (E)-1-(3-azidopropyl)4-(2-(7-(diethylamino)-2-oxo-2 $H$-chromen-3-yl)vinyl)pyridin-1ium $)^{28}$ (Fig. S1 and $\mathrm{S} 2 \dagger$ ). Tetrazine modified labeling reagents have advantage over other bioorthogonal motifs in that they react orders of magnitudes faster with their partners. ${ }^{23,29}$ The Cy3/Cy5 pair is widely used in FRET applications; ${ }^{14,18,26}$ on the contrary the other two fluorescent dye pairs are not available in the literature - according to the best of our knowledge. Almost identical overlaps were established for the Cy3T/Cy5T and $\mathrm{Cy} 1 \mathrm{~A} / \mathrm{Cy} 3 \mathrm{~T}$ pairs, while more considerable interaction is expected for the Cy1A/CBRD1A pair. CBRD1A is remarkable for its large Stokes-shift. ${ }^{28}$ Besides spectral overlap and distance, orientation of transition moments can also modulate the efficiency of energy transfer.

\section{Bioorthogonal fluorescent labeling of DNA oligomers}

In order to be able to test the selected partners quickly and efficiently, we aimed at establishing a concise protocol. To this end, we have selected complementary 17-mer oligonucleotide sequences for fluorescent derivatization. The selected sequences are suitable for the detection of Helicobacter pylori strains susceptible to clarithromycin (a macrolide antibiotic used in the first-line treatment of $H$. pylori) in gastric biopsies of human patients in FISH-based assays. ${ }^{30}$ The complementary oligos carried an amine modified linker either at the $5^{\prime}$ or $3^{\prime}$ end $\left(\mathrm{NH}_{2}-\left(\mathrm{CH}_{2}\right)_{6}{ }^{-{ }^{\prime}}{ }^{\prime}\right.$ CGG-GGT-CTT-TCC-GTC-TT ${ }^{3^{\prime}}-$ Clar $^{\mathbf{w t}} 5^{\prime}$; ${ }^{5^{\prime}}$ CGG-GGT-CTT-TCC-GTC-TT ${ }^{3^{\prime}}-\left(\mathrm{CH}_{2}\right)_{7}-\mathrm{NH} 2-\mathrm{Clar}^{\mathbf{w t}} \mathbf{3}^{\prime}$ and $\mathrm{NH}_{2}$ $\left(\mathrm{CH}_{2}\right)_{6}{ }^{{ }^{-}{ }^{\prime}}$ AAG-ACG-GAA-AGA-CCC-CG $\left.{ }^{3^{\prime}}-\mathbf{C l a r}^{\mathbf{w t}} \mathbf{K 5}^{\prime}\right)$. The oligos were modified with a strained cyclooctyne motif, $(1 R, 8 S, 9 S)$ bicyclo[6.1.0]non-4-yn-9-ylmethanol (BCN), via treatment of the amino modified DNA strands with commercially available BCN-succinimidyl ester (NHS-BCN) (Scheme 1). BCN was selected as a bioorthogonal motif as it can readily react with both azides and tetrazines in SPAAC and IEDDA reactions, respectively. In order to follow the installation of $\mathrm{BCN}$ onto the oligos and subsequent fluorescent labeling reactions a high resolution capillary zone electrophoretic (CE) method was developed (Fig. S3†). CE analysis indicated that the oligos, especially the GC rich $\mathbf{C l a r}^{\mathbf{w t}} \mathbf{5}^{\prime}$ and $\mathbf{C l a r}^{\mathbf{w t}} \mathbf{3}^{\prime}$, existed in multiple conformations probably due to inter- and intramolecular inter- 


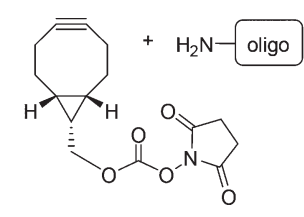

$\longrightarrow{ }_{\mathrm{H}}$ ${ }_{\mathrm{O}} \mathrm{II}_{\mathrm{NH}}$ oligo
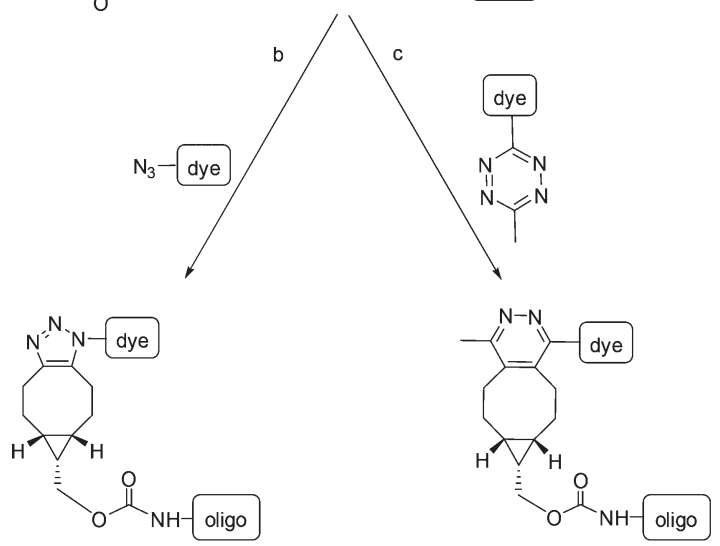

Scheme 1 Bioorthogonal labeling reactions of amino modified DNA oligomers. Derivatization with NHS-BCN; fluorescent labeling by an azide or tetrazine group harboring a dye through click chemistry. Experimental conditions: $100 \mu \mathrm{M}$ DNA oligo, $110 \mathrm{mM} \mathrm{NaHCO}_{3}+2.2 \mathrm{mM}$ EDTA buffer ( $\mathrm{pH}$ 9.0), incubation: $25^{\circ} \mathrm{C}$ for $20 \mathrm{~min}(\mathrm{a}, \mathrm{b}, \mathrm{c}), 5 \mathrm{mM}$ NHS-BCN (a), $500 \mu \mathrm{M}$ dye (b, c).

actions through H-bond formation giving rise to a group of peaks on the electropherogram. This finding is fully in line with secondary structure predictions using the mFOLD server, indicating the capacity to form an internal helix, incorporating the $5^{\prime}$ end of the oligonucleotide (Fig. S4 $\dagger$ ). The folding energy would correspond to an internal melting point of $\sim 41{ }^{\circ} \mathrm{C}$ at the ionic strength of $50 \mathrm{mM} \mathrm{Na}{ }^{+}$(the estimated melting temperature of the duplex is at $\sim 55{ }^{\circ} \mathrm{C}$ at the same ionic strength). As a consequence, some of these conformations turned out to be less reactive upon reaction with NHS-BCN. Elevation of the temperature to $60{ }^{\circ} \mathrm{C}$ for a short period of time $(5 \mathrm{~min})$ was found to be efficient to disrupt these secondary binding forces. As a result, a single peak appeared in the electropherogram. In addition, the terminal amino group became fully accessible; thus, upon treatment of $100 \mu \mathrm{M}$ amino-DNAs with 5 mM NHS-BCN nearly quantitative conversion (90\%) was obtained (Scheme 1). The efficiency of this CE method was quite satisfying as in each case the starting materials and the product could be well resolved. The reactions completed within 20 minutes in each case. A subsequent size exclusion (G25) spin column purification step was carried out yielding products of $90 \%$ purity with $20-25 \%$ loss. Subsequent fluorescent derivatization using fluorescent or fluorogenic dyes $(500 \mu \mathrm{M})$ required only $15 \mathrm{~min}$ incubation time at room temperature resulting in an average of $90 \%$ conversion. The product peaks were confirmed both by UV absorption and by fluorescent detection (LIF-CE) at wavelengths corresponding to the respective dyes. The products were purified similarly using a G25 spin column. The identification of the labeled oligos was carried out by LC-MS (Fig. S5-S15†). Final concentrations of the labeled oligos were estimated from the peak area in CE at $260 \mathrm{~nm}$ and also with UV detection using a Nanodrop system at $260 \mathrm{~nm}$. The total yields fell between 20 and $30 \%$ with $90 \%$ purity (Fig. S16-S26†).

\section{Application of fluorescently labeled DNA oligos as FISH probes}

Before moving onto the actual FRET studies, we were curious about the applicability of our labeled DNA strands in FISH applications. The selected oligomers are indeed suitable for the detection of Helicobacter pylori infections, through whole cell hybridization with the 23S rRNA sequence of $H$. pylori. We tested the suitability of two labeled oligos in the genotypic detection of clarithromycin resistance/susceptibility in H. pylori infected gastric tissues. ${ }^{30,31}$ Human gastric biopsies were treated with fluorescently (Cy3T) modified oligos Clar ${ }^{\mathbf{w t}} \mathbf{5}^{\prime}$ and Clar ${ }^{\text {wt }} \mathbf{3}^{\prime}$ (Fig. 2). We have chosen samples from patients suffering from clarithromycin-susceptible $H$. pylori infection (previously diagnosed by commercial FISH probes ${ }^{32}$ ) and healthy controls as well. In the microscopy images it is clearly seen that the bacteria fluoresce in red in the apical mucosa of the prefixed and treated sections, while no labeling can be detected in the healthy control samples (Fig. 2). Both the $5^{\prime}$ and $3^{\prime}$ conjugated DNA strands seemed similarly efficient in this FISH protocol. These results suggest that suitable fluorescent dyes, even ones not available commercially can be easily adopted for FISH applications using the above protocol.

\section{FRET models}

Fluorescently labeled complementary oligonucleotide strands provide an easy tool for investigating the suitability of novel dyes in FRET application. ${ }^{33,34}$ When fluorescence modifications were situated in close proximity ( $5^{\prime}$ and $3^{\prime}$ matched) the estimated distance of the dyes was $18 \AA$; while positioned at distal ends ( $5^{\prime}$ and $5^{\prime}$ matched sequences) this distance was

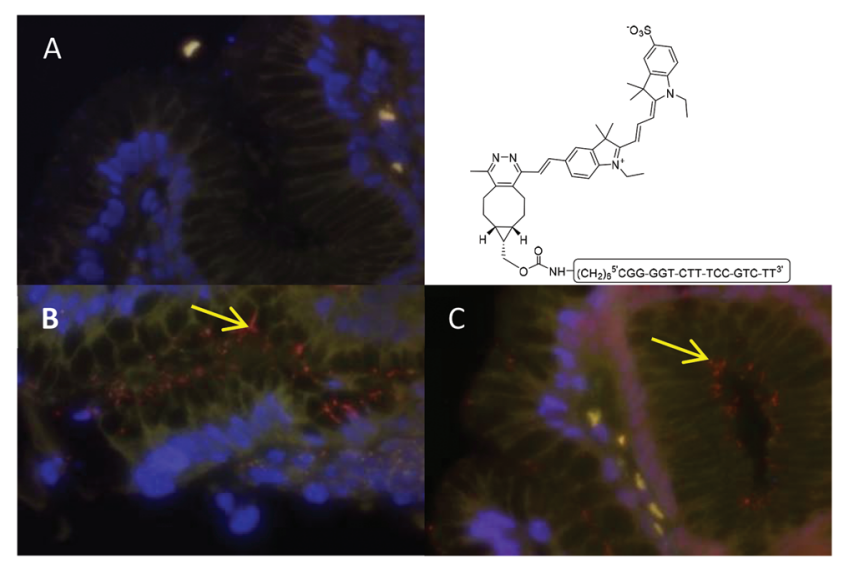

Fig. 2 Labeling of clarithromycin sensitive Helicobacter pylori by a Cy3T modified DNA oligomer in gastric biopsies of healthy (A) and infected $(B, C)$ patients. The arrows indicate that the stained bacteria fluoresce in red in the mucosa. The DAPI nuclear counterstain produces blue fluorescence (original magnification: $63 \times$ ). Labeling efficiency was independent of the modification site $\left(3^{\prime}\right.$ or $5^{\prime}$, Panels $B$ and $C$, respectively). 

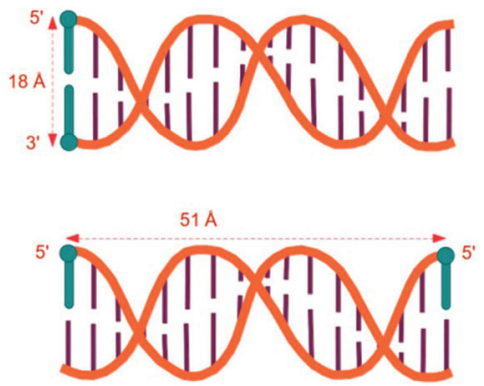

Fig. 3 Estimated distances of the 17-base pair long double stranded DNA.

$51 \AA \pm 5 \AA$ (Fig. 3 and Table S1†). Since in FRET 50\% efficiency can be achieved at around these distances according to the literature data the predicted lengths for oligomer pairs were quite suitable for the estimation of Förster radii (e.g. for Cy3-Cy5 pairs Förster radius $R_{0}=56-59 \AA$ ).$^{13,27,35}$

We prepared all combinations of fluorescently labeled DNA oligos by the above two-step method. Since FRET efficiency seemed to be independent of the labeling site (i.e. which strand was conjugated by the acceptor or donor dyes) we show here the case where $\operatorname{Clar}^{\mathbf{w t}} \mathbf{3}^{\prime}$ and $\mathbf{C l a r}^{\mathbf{w t}} \mathbf{5}^{\prime}$ were modified with the acceptor dyes while the counterstrand $\mathbf{C l a r}^{\mathbf{w t}} \mathbf{K} 5^{\prime}$ held the donors. The spectral overlap of the fluorescently labeled DNA oligomers is presented in Fig. 4.

\section{Testing the FRET efficiencies of selected dye pairs}

FRET efficiencies were monitored by measuring fluorescence intensities and changes in the spectra of the donor and the acceptor peaks. Before analyzing the results obtained upon annealing the oligomers we aimed to assess the occurrence of any non-specific FRET signals. The concentration range of the DNAs in FRET measurements was chosen according to the dissociation constant of the strands $(1-10 \mathrm{nM}) .{ }^{36}$ At $500 \mathrm{nM}$ with a 1:1 ratio of the free dyes (not conjugated to DNA) no FRET signal could be observed. Furthermore, at the same concentration, the acceptor-oligo conjugates did not demonstrate any remarkable fluorescent signal when excited at the excitation wavelength of the respective donor.

Fig. 5 shows the changes in normalized fluorescence intensities of the donor and acceptor dyes, when the concentration of the donor-Clar ${ }^{\mathbf{w t}} \mathbf{K} \mathbf{5}^{\prime}$ was kept constant (500 nM), while the concentration of the acceptor-Clar ${ }^{\text {wt }} \mathbf{3}^{\prime}$ or $\mathbf{C l a r}^{\mathbf{w t}} \mathbf{5}^{\prime}$ varied within the range of 62.5 to $2000 \mathrm{nM}$. The maximal FRET efficiencies are summarized in Table 1. The apparent FRET efficiencies were calculated according to the following equation: $E_{\mathrm{app}}=1-\left(I_{\mathrm{DA}} / I_{\mathrm{D}}\right)$, where $I_{\mathrm{DA}}$ and $I_{\mathrm{D}}$ are the fluorescence intensity of the donor dye measured in the presence and in the absence of the acceptor, respectively. $E_{\text {app }}$ provides only an approximate indicator of the dye-dye distances because of the uncertainty of the orientation factor of the fluorophores. Furthermore, instrumental corrections were ignored and are not involved in the equation.

Regarding proximal modification - i.e. annealing of labeled Clar $^{\mathbf{w t}} \mathbf{3}^{\prime}$ and Clar $^{\mathbf{w t}} \mathbf{K} \mathbf{5}^{\prime}$ - the most impressive decrease in the
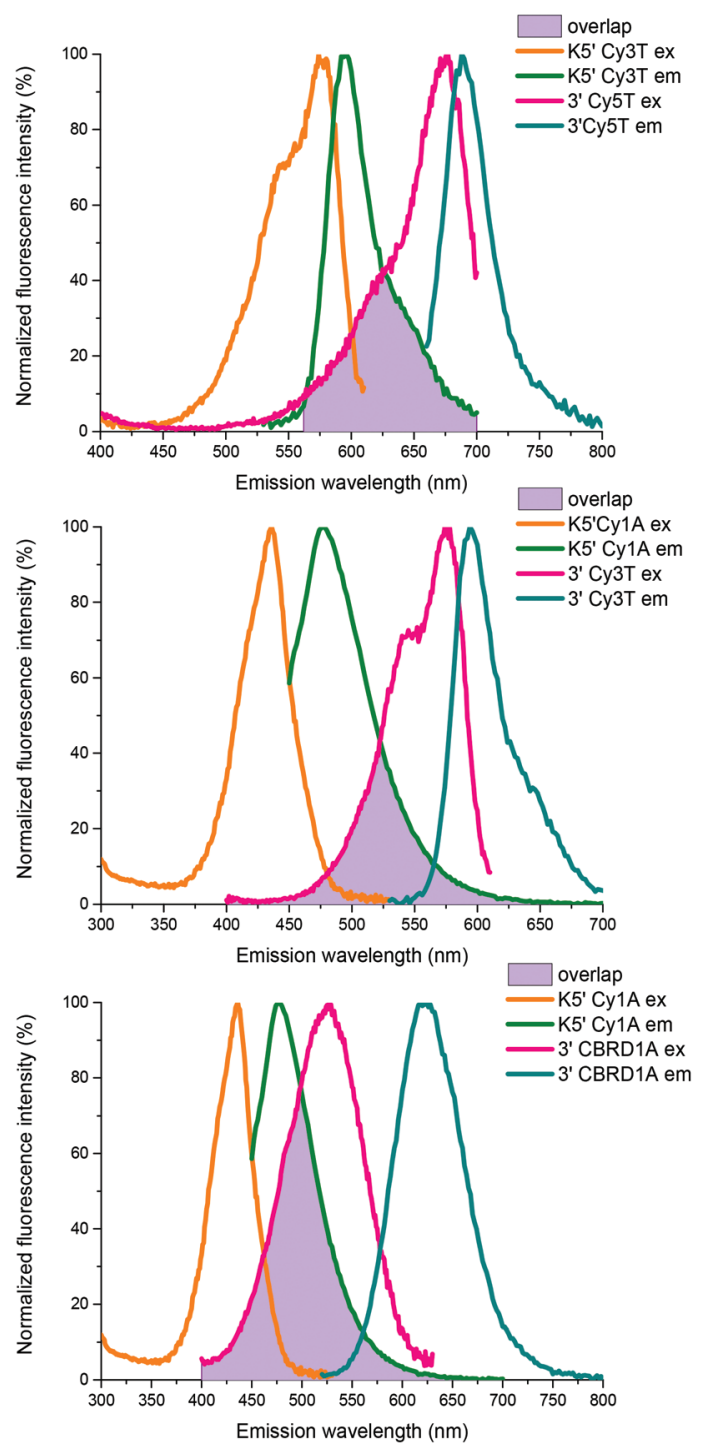

Fig. 4 Spectral overlap of the DNA oligomers labeled by the selected dye pairs.

donor fluorescence was detected for the Cy3T/Cy5T pair (nearly 90\%). In the case of the other two pairs the decrease in the donor fluorescence was $c a$. 70\%. On analysing the increase in the acceptor fluorescence intensity a negligible effect for Cy3T/Cy5T was observed. On the contrary, in the case of the Cy1A/Cy3T and Cy1A/CBRD1A pairs, acceptor signals changed considerably upon annealing. The specificity of the FRET signals was further supported by competition studies using unlabeled strands. Increasing amounts of unlabeled competitor strands resulted in a substantial decrease of the FRET signal (data not shown).

When fluorescence modifications were localized at the distal termini of the DNA oligomers, FRET efficiencies dropped in general and only 39-56\% FRET efficiencies could be achieved. On the other hand quite a good estimation of the Förster radii could be done suggesting that $R_{0}$ is approximately $50 \AA$ for the Cy1A/CBRD1A pair and a bit shorter for the other 

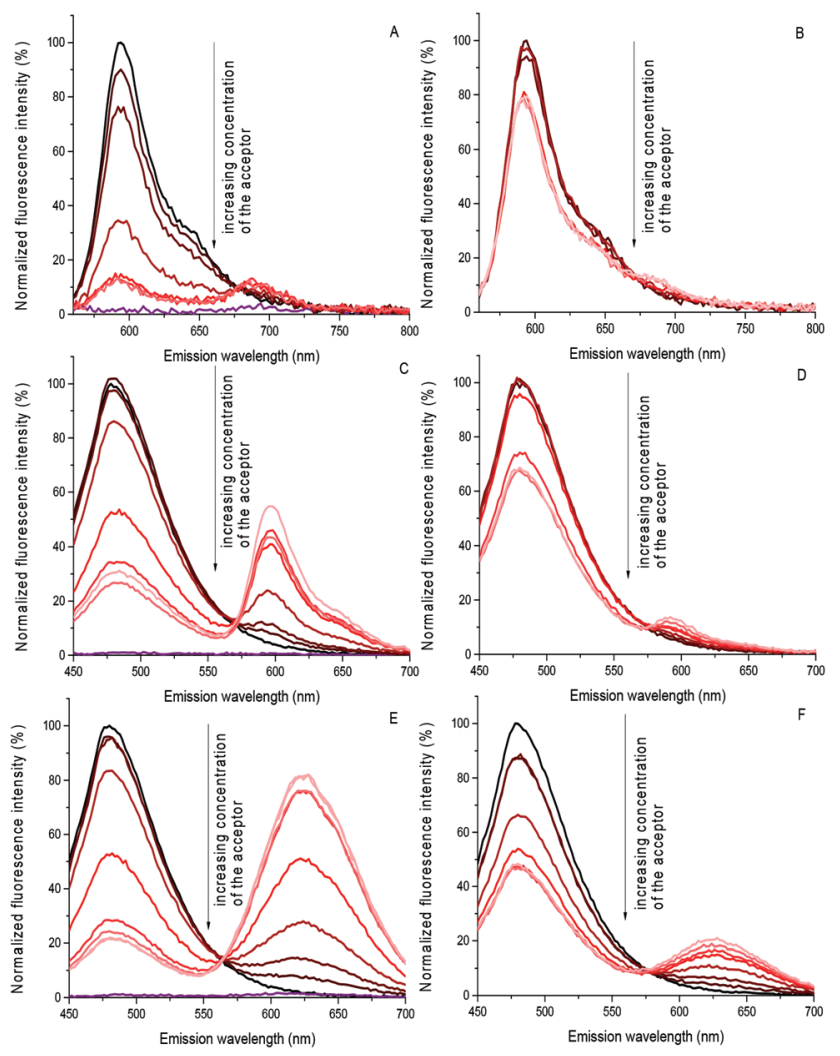

Fig. 5 Changes in fluorescence intensity of the donor-DNA conjugate (500 nM; control-black) upon increasing acceptor concentration (62.5-2000 nM from brown to red). Fluorescence spectra of the acceptor-DNA conjugate $(500 \mathrm{nM})$ are presented in violet $(\mathrm{A}, \mathrm{C}$, and $\mathrm{E}) . \mathrm{A}, \mathrm{C}$, $E$ : fluorescent labeling at the proximal termini of the DNA oligomers; $B$, $D$, F: fluorescent labeling at the distal termini of the DNA oligomers; A-B: Cy3T/Cy5T; C-D: Cy1A/Cy3T; E-F: Cy1A/CBRD1A; A, B: $\lambda_{\text {ex }}: 540 \mathrm{~nm}$; C, D, E, F: $\lambda_{\text {ex }}: 420 \mathrm{~nm}$.

Table 1 Maximal FRET efficiencies and melting points of labeled DNA oligomer pairs

\begin{tabular}{llll}
\hline Dye pairs & Labeling site & FRET efficiency $^{a}(\%)$ & $T_{\mathrm{m}}{ }^{b}\left({ }^{\circ} \mathrm{C}\right)$ \\
\hline Cy3T/Cy5T & Proximal & 92 & 65 \\
& Distal & 39 & 57 \\
Cy1A/Cy3T & Proximal & 70 & 61 \\
& Distal & 40 & 57 \\
Cy1A/CBRD1A & Proximal & 77 & 63 \\
& Distal & 56 & 59
\end{tabular}

${ }^{a}$ FRET efficiencies were observed at $500 \mathrm{nM}$ donor-Clar ${ }^{\mathbf{w t}} \mathbf{K} \mathbf{5}^{\prime}$ conjugate and labeled Clar ${ }^{\text {wt }} 3^{\prime}$ and Clar ${ }^{\text {wt }^{\prime}}{ }^{\prime}$ from 62.5 to $2000 \mathrm{nM}$. ${ }^{b}$ Melting points $\left(T_{\mathrm{m}}\right)$ were estimated from the FRET efficiency changes upon temperature controlled denaturation of the complementary DNA oligomer pairs.

two pairs. The specificity of FRET signals was further tested by the temperature controlled denaturation of the hybridized double stranded DNA oligomers. Changes in the fluorescence spectra were monitored between $25^{\circ} \mathrm{C}$ and $75^{\circ} \mathrm{C}$. Data were corrected with the temperature dependent fluorescence changes of single stranded oligos. Around the melting point
$\left(T_{\mathrm{m}}=55-60{ }^{\circ} \mathrm{C}\right)$ the FRET efficiencies dramatically dropped both in the case of the proximally and distally labeled oligonucleotide pairs (Fig. S27†). The apparent melting point shifted to higher values in the case of proximally labeled strands, suggesting the existence of attractive interactions between the dyes (Table 1).

\section{FRET signals in live cells}

DNA oligomers labeled with Cy1A and Cy3T dye pairs were selected for the microscopy analysis of FRET signals in live cells. HEK293T cells were transfected with the donor (Cy1A conjugated $\mathbf{C l a r}^{\mathbf{w t}} \mathbf{K} 5^{\prime}$ ) or with the acceptor (Cy3T conjugated Clar $^{\text {wt }} 3^{\prime}$ ) or simultaneously with both labeled oligomers. Transfection efficiencies were monitored by flow cytometry

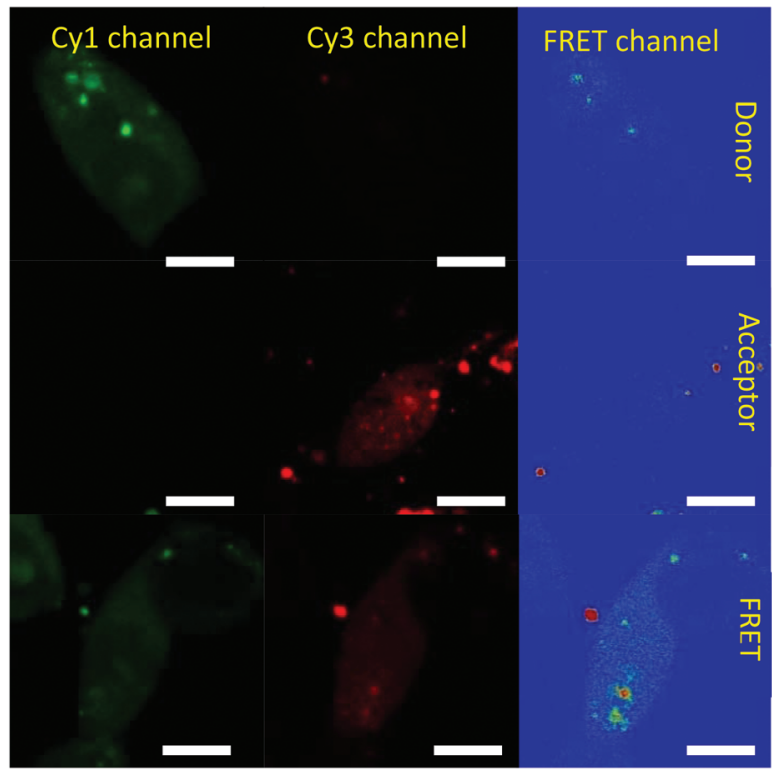

Fig. 6 Fluorescence imaging of HEK293T cells transfected with oligos labeled either with the donor (Cy1A conjugated $\mathrm{Clar}^{\mathrm{Wt}} \mathrm{K5}^{\prime}$; top row) or the acceptor (Сy3T conjugated Clar ${ }^{\text {wt }} 3^{\prime}$ : middle row) or both (bottom row) detected in the Cy1 channel $\left(\lambda_{\mathrm{ex}}: 405 \mathrm{~nm} ; \lambda_{\mathrm{em}}\right.$ : 475-545 nm); Cy3 channel ( $\lambda_{\mathrm{ex}}: 514 \mathrm{~nm} ; \lambda_{\mathrm{em}}: 620-800 \mathrm{~nm}$ ); and FRET channel $\left(\lambda_{\mathrm{ex}}: 405 \mathrm{~nm} ; \lambda_{\mathrm{em}}: 620-800 \mathrm{~nm}\right)$. White bars represent $10 \mu \mathrm{m}$.

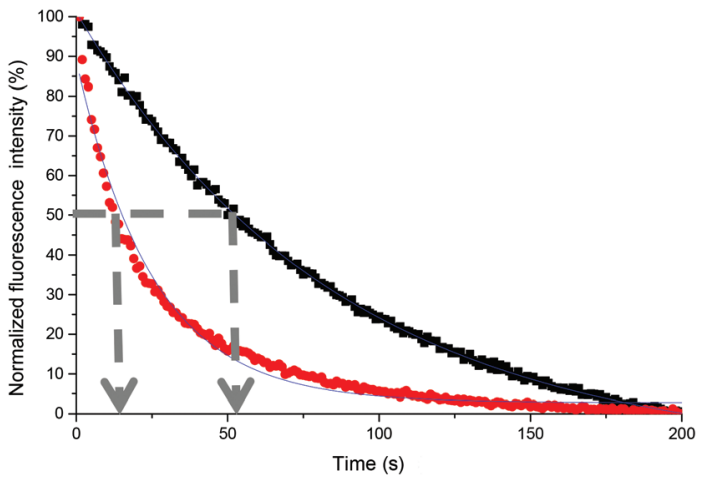

Fig. 7 Difference in bleaching kinetics of the donor in the presence (black) and in the absence (red) of the acceptor. 
(Fig. S28†). Confocal fluorescence microscopy images indicate that there is no cross-talk between the dyes and no emission of the acceptor occurs (between 620 and $800 \mathrm{~nm}$ ) upon excitation at the donor's excitation wavelength $(405 \mathrm{~nm})$. On the other hand, when the cells were transfected with both the donor and the acceptor, evident FRET signals could be detected between 620 and $800 \mathrm{~nm}$ upon excitation at $405 \mathrm{~nm}$ (Fig. 6). The bleaching kinetics was also analyzed. Fluorescence intensity of the donor dropped to half of the original value within $13 \mathrm{~s}$ in the absence of the acceptor, while an extended time course of $50 \mathrm{~s}$ was observed in the presence of the acceptor, further confirming the efficiency of FRET (Fig. 7).

\section{Conclusions}

We designed bioorthogonalized oligonucleotide strands based on previously reported Clar ${ }^{\text {wt }}$ sequences as platforms suitable for fast and efficient labeling by azide or tetrazine modified dyes. Not only are the selected sequences suitable for the elaboration of FRET efficiencies, the length of the oligonucleotides also allows for the estimation of Förster radii.

We have used these platforms to test selected, bioorthogonally applicable fluorescent probes from our existing dye-set. The results indicated that the selected dyes exhibit strong FRET signals (70-90\%) when the dyes are situated at proximal termini. As expected, decreased FRET efficiencies were detected when the distal termini were labeled. We have also examined the changes in FRET efficiencies as a function of temperature and concluded that in case of the double stranded oligos labeled at the proximal ends the $T_{\mathrm{m}}$ shifted towards higher temperatures, presumably due to attractive interactions between the dyes. The specificity of the FRET signals was affirmed by competitive studies using a non-labeled counterstrand. Regarding the pairs tested, the use of the Cy3T/Cy5T pairs is proposed when decrease in fluorescence intensity of the donor dye is desirable upon interactions between macromolecules, while the Cy1A/CBRD1A or Cy1A/Cy3T pairs are suggested as primary choices when evolution of the acceptor signal is to be detected. The Cy1A/Cy3T pairs were further tested in live cells. Confocal microscopy images showed no considerable cross-talk between the donor and the acceptor, while a remarkable FRET signal was observed in HEK293T cells transfected with complementary DNA oligomers labeled fluorescently with the donor and the acceptor dyes, respectively. The evolution of the FRET effect was further proved by the donor bleaching kinetics in the presence of the acceptor. Furthermore, the Cy3T labeled Clar ${ }^{\text {wt }}$ oligomers were applied in the FISH detection of $H$. pylori in gastric biopsy samples showing further use of the two-step - bioorthogonalization and bioorthogonal labeling - modification scheme. We believe that such easily accessible and modifiable oligonucleotide platforms can be applied in screening for further spectrally matching fluorophore pairs. The newly identified FRET pairs are suitable for expanding the spectral range in FRET applications, not to mention the fact that these dyes are easily installed to virtually any kind of biomolecules following bioorthogonalization.

\section{Experimental}

\section{General methods}

Unless otherwise indicated, all starting materials were obtained from commercial suppliers (Sigma-Aldrich, Fluka, Merck, Alfa Aesar, Reanal, Molar Chemicals, and Fluorochem) and used without further purification. Analytical thin-layer chromatography (TLC) was performed on silica gel 60 F254 precoated aluminium TLC plates from Merck. Visualization of TLC samples was performed with a 254/365 nm UV lamp. NMR spectra were recorded on a Varian Inova $500 \mathrm{MHz}$ instrument. Chemical shifts $(\delta)$ are given in parts per million (ppm) using solvent signals as the reference. Coupling constants $(J)$ are reported in hertz $(\mathrm{Hz})$. The exact masses were determined with an Agilent 6230 time-of-flight mass spectrometer. All melting points were measured on an OptiMelt Automated Melting Point System and are uncorrected.

\section{Synthesis}

The synthesis of CBRD1A has been described previously by Kele et $a .^{28}$ The synthesis and application of Cy3T and Cy5T is published elsewhere. ${ }^{37}$

\section{Synthesis of Cy1-azide}

2-((6-Azido-3-ethyl-1,3-benzothiazol-2(3H)-ylidene)methyl)-3ethyl-1,3-benzothiazol-3-ium hexafluorophosphate. A mixture of 3-ethyl-2-methylbenzothiazol-3-ium iodide $^{38}(100 \mathrm{mg}$, $0.33 \mathrm{mmol}$ ), 6-azido-3-ethyl-2-methylsulfanylbenzothiazolium sulfate $^{39}$ (108 mg, $\left.0.36 \mathrm{mmol}\right)$ and triethylamine $(50 \mu \mathrm{L}$, $0.36 \mathrm{mmol}$ ) in $10 \mathrm{~mL}$ ethanol was stirred at $75^{\circ} \mathrm{C}$ for $60 \mathrm{~min}$ under a $\mathrm{N}_{2}$ atmosphere (Scheme 2). After cooling, the solid was filtered and washed with cold ethanol. The salt was dissolved in acetonitrile, and $0.3 \mathrm{~g}$ ammonium-hexafluorophosphate in water was added to the solution. The acetonitrile was removed, and the precipitated product was filtered, washed with water and dried in vacuo to give Cy1A $(57 \mathrm{mg}$, $0.108 \mathrm{mmol}, 33 \%$ ) as a yellow solid. The product was used without further purification. $R_{\mathrm{f}}: 0.51$ (cc. $\mathrm{NaCl}: \mathrm{EtOH}=1: 9$ ); m.p.: decomp. in the presence of light; ${ }^{1} \mathrm{H}$ NMR $(500 \mathrm{MHz}$, DMSO-d $\left._{6}\right) \delta 8.25(\mathrm{~d}, J=7.7 \mathrm{~Hz}, 1 \mathrm{H}), 8.10(\mathrm{~d}, J=2.1 \mathrm{~Hz}, 1 \mathrm{H})$, $7.91(\mathrm{~d}, J=8.7 \mathrm{~Hz}, 2 \mathrm{H}), 7.70(\mathrm{t}, J=7.4 \mathrm{~Hz}, 1 \mathrm{H}), 7.51(\mathrm{t}, J=7.6$

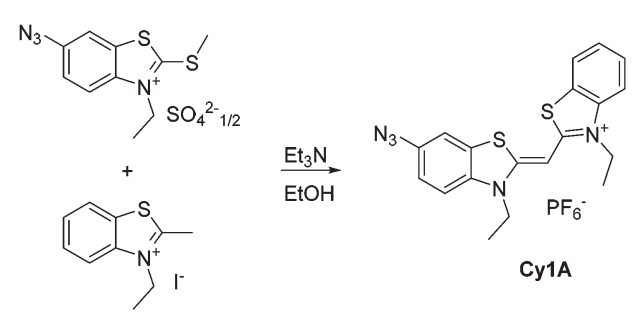

Scheme 2 Synthesis of the Cy1A dye. 
$\mathrm{Hz}, 1 \mathrm{H}), 7.43$ (dd, $J=8.8,2.0 \mathrm{~Hz}, 1 \mathrm{H}), 6.72(\mathrm{~s}, 1 \mathrm{H}), 4.73-4.63$ $(\mathrm{m}, 4 \mathrm{H}), 1.40(\mathrm{t}, J=7.3 \mathrm{~Hz}, 3 \mathrm{H}), 1.39(\mathrm{t}, J=7.3 \mathrm{~Hz}, 3 \mathrm{H}) ;{ }^{13} \mathrm{C}$ NMR (126 MHz, DMSO-d $\left.{ }_{6}\right) \delta 161.3,161.2,139.8,137.2,136.6$, $128.7,126.8,125.0,125.0,123.6,120.1,114.7,113.9,113.6$, 82.1, 41.8, 41.6, 12.2; HRMS: $[\mathrm{M}]^{+}$: calcd for $\left[\mathrm{C}_{19} \mathrm{H}_{18} \mathrm{~N}_{5} \mathrm{~S}_{2}\right]^{+}$: 380.1004 , found: 380.1002 . For details see the ESI. $\dagger$

\section{DNA oligomers}

The DNA oligos $\left(\mathrm{NH}_{2}-\left(\mathrm{CH}_{2}\right)_{6}{ }^{-{ }^{\prime} \mathrm{CGG}-G G T-C T T-T C C-G T C-T T^{3}}{ }^{\prime}\right.$, ${ }^{5}$ CGG-GGT-CTT-TCC-GTC-TT ${ }^{3^{\prime}}-\left(\mathrm{CH}_{2}\right)_{7}-\mathrm{NH}_{2}$ and $\mathrm{NH}_{2}-\left(\mathrm{CH}_{2}\right)_{6}{ }^{-5^{\prime}}$ AAG-ACG-GAA-AGA-CCC-CG ${ }^{3}$ ) purchased from Metabion (Steinkirchen, Germany) were diluted in sterile distilled water in $1 \mathrm{mM}$ concentration. These stock solutions were further diluted ten times with $110 \mathrm{mM} \mathrm{Na} \mathrm{CO}_{3}-\mathrm{NaHCO}_{3}$, buffer ( $\mathrm{pH}$ 9.0) containing 2.2 mM EDTA.

Based on several, unrelated, experimentally determined DNA or DNA-protein complexes available in the Protein Data Bank (PDB), the average distance between two opposing monomers that are 17 positions apart was estimated (Table $\mathrm{S} 1 \dagger$ ). The average distance was not altered by including mildly bent, distorted, crosslinked or damaged DNA duplexes, as long as strands were fully complementary.

\section{Conjugation processes}

DNA oligos $(100 \mu \mathrm{M})$ in $110 \mathrm{mM} \mathrm{NaHCO}{ }_{3}+2.2 \mathrm{mM}$ EDTA buffer pH 9.0 were incubated at $60{ }^{\circ} \mathrm{C}$ for five minutes before labeling. (1R,8S,9S)-Bicyclo[6.1.0]non-4-yn-9-ylmethyl $\mathrm{N}$-succinimidyl ester (NHS-BCN) was added to the oligos in $5 \mathrm{mM}$ concentration at room temperature for $20 \mathrm{~min}$. The modified oligos were separated from the excess of NHS-BCN on a Sephadex G25 "Fine" desalting gel (Pharmacia Fine Chemicals, Sweden) with SpinPrep column technology (Sigma, St Louis, MO, USA). The modified oligos were fluorescently labeled with $500 \mu \mathrm{M}$ dyes (Cy1A, Cy3T, Cy5T and CBRD1A) at room temperature for $20 \mathrm{~min}$. The samples were purified with the desalting G25 spin column described above.

\section{Analytical methods}

Capillary electrophoresis. The background electrolyte (BGE) component boric acid was purchased from Sigma (St Louis, MO, USA), and sodium hydroxide, sodium carbonate and bicarbonate were purchased from Merck GmbH (Darmstadt, Germany).

Capillary electrophoresis was performed with an Agilent Capillary Electrophoresis $3 \mathrm{D}^{\mathrm{CE}}$ system (Agilent Technologies, Waldbronn, Germany) applying a bare fused silica capillary having $64.5 \mathrm{~cm}$ total and $56 \mathrm{~cm}$ effective length with $50 \mu \mathrm{m}$ I.D. (Agilent Technologies, Santa Clara, CA, USA). On-line absorption at $260 \mathrm{~nm}$ was monitored for the detection of DNA and $420 \mathrm{~nm}, 500 \mathrm{~nm}, 540 \mathrm{~nm}$ and $595 \mathrm{~nm}$ for Cy1A, CBRD1A, Cy3T and Cy5T, respectively by a DAD UV-Vis detector. A laser $\left(\lambda_{\text {ex }}: 488 \mathrm{~nm}\right)$ (Melles Griot, Carlsbad, USA) induced fluorescent (LIF) signal was observed by a ZetaLIF Discovery detector (Picometrics, Labege, France) at $45.5 \mathrm{~cm}$ effective length. The capillary was thermostated at $25{ }^{\circ} \mathrm{C}$. Between measurements, the capillary was rinsed subsequently with $0.1 \mathrm{M} \mathrm{HCl}, 1.0 \mathrm{M}$
$\mathrm{NaOH}, 0.1 \mathrm{M} \mathrm{NaOH}$ and distilled water for 3 minutes each and with BGE (200 mM sodium borate ( $\mathrm{pH}$ 9.0)) for 5 minutes. Samples were injected by $5 \times 10^{3}$ Pa pressure for 6 s. Runs were performed in the positive-polarity mode with $30 \mathrm{kV}$.

Nanodrop. Final concentrations of the dye-DNA conjugates were determined based on UV absorbance at $260 \mathrm{~nm}$ using a Nanodrop ND-1000 V3.5.2 spectrophotometer (NanoDrop Technologies, Wilmington, USA). A $3 \mu$ l sample was dropped onto the detector cell.

LC-MS analyses of fluorescently labeled oligonucleotides. A Sciex 6500 QTRAP triple quadrupole/linear ion trap mass spectrometer, equipped with a Turbo V Source in an electrospray mode (Sciex, CA, USA), and a PerkinElmer Series 200 micro LC system (Massachusetts, USA) consisting of a binary pump and an autosampler were used for LC-MS analysis. Data acquisition and processing were performed using Analyst software version 1.6.2 (AB Sciex Instruments, CA, USA). Chromatographic separation was achieved by a Purospher STAR RP-18 endcapped $(55 \mathrm{~mm} \times 2.1 \mathrm{~mm}, 3 \mu \mathrm{m})$ LiChocart ${ }^{\circledR}$ 55-2 HPLC cartridge. The sample was eluted with gradient elution using solvent A ( $0.1 \mathrm{mM}$ ammonium formate in water) and solvent $\mathrm{B}(0.1 \mathrm{mM}$ ammonium formate in $\mathrm{MeOH})$. The

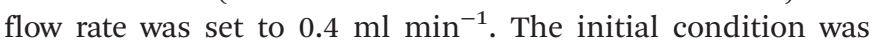
$10 \% \mathrm{~B}$ for $1 \mathrm{~min}$, followed by a linear gradient to $90 \% \mathrm{~B}$ by $5 \mathrm{~min}$; from 6 to $8 \mathrm{~min} 90 \% \mathrm{~B}$ was retained; and from 8 to 8.5 min it was back to the initial condition with $10 \%$ eluent B and retained to $13 \mathrm{~min}$. The column temperature was maintained at room temperature and the injection volume was $10 \mu \mathrm{l}$ (200-300 pmol on column). Nitrogen was used as the nebulizer gas (GS1), heater gas (GS2), and curtain gas with the optimum values set at 35, 40 and 40 (arbitrary units). The data were acquired in a negative ionization mode from $\mathrm{m} / \mathrm{z}=300$ to 2000 in $1.7 \mathrm{~s}$. The source temperature was $400{ }^{\circ} \mathrm{C}$ and the spray voltage was set at $-4500 \mathrm{~V}$. The declustering potential value was set to $-60 \mathrm{~V}$.

\section{Fluorescence in situ hybridization}

Clarithromycin-susceptible Helicobacter pylori bacteria were labeled by our fluorescently modified (Cy3T) oligos in human samples as described earlier. ${ }^{30,32}$ In short, a freshly cut $4 \mu \mathrm{m}$ tissue section of the formalin-fixed, paraffin-embedded gastric mucosa specimen was mounted on a coated glass slide, deparaffinized and microwaved in a Vector Antigen Unmasking Solution H-3300 (Vector Laboratories, Burlingame, CA, USA) at $400 \mathrm{~W}$ for 10 minutes. Hybridization solution containing one of the fluorescently (Cy3T) modified oligos (Clar ${ }^{\mathbf{w t}} \mathbf{5}^{\prime}$ and Clar $^{\text {wt }} 3^{\prime}$ ) was added to the specimens and incubated for 90 minutes at $46{ }^{\circ} \mathrm{C}$. After a post-hybridization wash (15 minutes at $46{ }^{\circ} \mathrm{C}$ ), the slides were mounted using a fluorescent mounting medium containing a blue fluorescent nuclear counterstain (Vectashield with DAPI, Vector Laboratories, Burlingame, CA, USA). Specific signals were examined using an epifluorescence microscope Leica DM-RXA (Leica Microsystems, Wetzlar, Germany) equipped with a DAPI filter for blue fluorescence as well as Spectrum Green and Spectrum Orange filters (Vysis, Downers Grove, IL, USA) for 
green and orange-red fluorescence, respectively. Images were taken using a Leica DFC365 FX camera and documented by Leica CW4000 FISH analysis software.

\section{FRET measurements}

Fluorescence spectra were obtained by a PerkinElmer EnSpire Multimode Reader (PerkinElmer Inc.; Waltham; USA), a plate reader using a 384 well round bottom black plate (Corning; Corning; NY, USA) at room temperature with 100 flashes per point. The sample volume was $20 \mu \mathrm{l}$. Data were acquired and handled by Corning Enspire Manager 4.10 software. Excitation wavelengths were $420 \mathrm{~nm}$ and $560 \mathrm{~nm}$ for Cy1A and Cy3T, respectively.

The temperature dependency of FRET efficiency was investigated using a Jasco FP-8300 spectrofluorimeter with the Spectra Manager 2.12 software between 25 and $75{ }^{\circ} \mathrm{C}$. A heating step of $2{ }^{\circ} \mathrm{C}$ was achieved by elevating $0.8{ }^{\circ} \mathrm{C} \mathrm{min}^{-1}$, and incubating for $2 \mathrm{~min}$. A $200 \mu \mathrm{l}$ sample was measured in a QS High Precision Cell, a $10 \mathrm{~mm}$ narrow SUPRASIL ${ }^{\circledR}$ quartz cuvette (HellmaAnalytics, Germany).

\section{Cell culture}

HEK293T cells (ATCC® CRL3216 ${ }^{\mathrm{TM}}$ ) were cultured in Dulbecco's modified Eagle's medium (DMEM) (Life Technologies 41965-039) completed with 1\% penicillinstreptomycin (Sigma P0781), 1\% L-glutamine (Sigma G7513), $1 \%$ sodium pyruvate (Life Technologies 11360), and 10\% FBS (Sigma F7524) at $37{ }^{\circ} \mathrm{C}$ under a $5 \% \mathrm{CO}_{2}$ atmosphere.

\section{FRET microscopy}

For imaging we used poly-L-lysine treated 8-well Lab-Tek II chambers (Lab-Tek 155409). The wells were pretreated with $0.01 \mathrm{mg} \mathrm{ml}^{-1}$ poly-L-lysine (Sigma P5899) for $4 \mathrm{~h}$ at room temperature and washed twice with PBS. HEK293T cells were seeded into the chambers (30000 cell per well) and incubated for $24 \mathrm{~h}$ at $37{ }^{\circ} \mathrm{C}$ under a $5 \% \mathrm{CO}_{2}$ atmosphere. Afterwards the cells were transfected with 25 pmol fluorescently labeled DNA using Lipofectamine-3000 (Invitrogen L3000-001) and Optimem medium (Gibco 70011-044) and incubated for $4 \mathrm{~h}$ at $37{ }^{\circ} \mathrm{C}$ under a $5 \% \mathrm{CO}_{2}$ atmosphere. Before measurements, cells were washed with PBS. Imaging was performed on a Zeiss LSM 710 microscope, applying a Zeiss PlanApo 40× (NA 1.4) objective. Bleaching constants were calculated by Fiji software and the exponential decay equation was calculated using Origin Pro9 software (OriginLab Corp.).

\section{Conflicts of interest}

There are no conflicts to declare.

\section{Acknowledgements}

György Várady and Edit Szabó are greatly acknowledged for the flow cytometric measurements and Erzsébet Kovács for the technical support in FISH analysis. The research was financially supported by the Hungarian Academy of Sciences and the Hungarian Scientific Research Fund (Lendület Grant - LP 2013-55/2013 and OTKA-NN-116265). G. K. and É. K. acknowledge the support of the ÚNKP-17-3 New National Excellence Program of the Ministry of Human Capacities (ÚNKP-17-3-IV-ELTE-274 and ÚNKP_17-3-II-SE-3).

\section{References}

1 Y. Yan, Curr. Opin. Chem. Biol., 2003, 7, 635.

2 A. Miyawaki, Dev. Cell, 2003, 4, 295.

3 V. Marx, Nat. Methods, 2017, 14, 949.

4 X. Chen and Y. W. Wu, Org. Biomol. Chem., 2016, 14, 5417.

5 K. Rombouts, K. Braeckmans and K. Remaut, Bioconjugate Chem., 2016, 27, 280.

6 E. Kozma, O. Demeter and P. Kele, ChemBioChem, 2017, 18, 486.

7 V. V. Didenko, BioTechniques, 2001, 31, 1106.

8 C. Cui, W. Shu and P. Li, Front. Cell Dev. Biol., 2016, 4, 89.

9 L. Hu, K. Ru, L. Zhang, Y. Huang, X. Zhu, H. Liu, A. Zetterberg, T. Cheng and W. Miao, Biomark. Res., 2014, $2,3$.

10 G. Ramsay, Nat. Biotechnol., 1998, 16, 40.

11 R. Bumgarner, Curr. Protoc. Mol. Biol, 2013, Chapter 22, Unit 221.

12 X. P. He, X. L. Hu, T. D. James, J. Yoon and H. Tian, Chem. Soc. Rev., 2017, 46, 6687.

13 P. G. Wu and L. Brand, Anal. Biochem., 1994, 218, 1.

14 H. Sahoo, J. Photochem. Photobiol., C, 2011, 12, 20.

15 S. E. Braslavsky, E. Fron, H. B. Rodriguez, E. S. Roman, G. D. Scholes, G. Schweitzer, B. Valeur and J. Wirz, Photochem. Photobiol. Sci., 2008, 7, 1444.

16 T. Förster, Fluoreszenz organischer Verbindungen, Vandenhoeck \& Ruprecht, Göttingen, 1951.

17 L. M. Loura, Int. J. Mol. Sci., 2012, 13, 15252.

18 A. Munoz-Losa, C. Curutchet, B. P. Krueger, L. R. Hartsell and B. Mennucci, Biophys. J., 2009, 96, 4779.

19 I. Täpp, L. Malmberg, E. Rennel, M. Wik and A. C. Syvänen, BioTechniques, 2000, 28, 732.

20 S. Tyagi and F. R. Kramer, F1000 Med. Rep., 2012, 4, 10.

21 Y. J. Chen, B. Groves, R. A. Muscat and G. Seelig, Nat. Nanotechnol., 2015, 10, 748.

22 N. Xie, J. Huang, X. Yang, X. He, J. Liu, J. Huang, H. Fang and K. Wang, Anal. Chem., 2017, 89, 12115.

23 G. B. Cserep, A. Herner and P. Kele, Methods Appl. Fluoresc., 2015, 3, 042001.

24 P. Z. Qin and A. M. Pyle, Methods, 1999, 18, 60.

25 M. Merkel, S. Arndt, D. Ploschik, G. B. Cserep, U. Wenge, P. Kele and H. A. Wagenknecht, J. Org. Chem., 2016, 81, 7527.

26 A. Dietrich, V. Buschmann, C. Müller and M. Sauer, Rev. Mol. Biotechnol., 2002, 82, 211.

27 R. A. Cardullo, S. Agrawal, C. Flores, P. C. Zamecnik and D. E. Wolf, Proc. Natl. Acad. Sci. U. S. A., 1988, 85, 8790. 
28 P. Kele, G. Mezo, D. Achatz and O. S. Wolfbeis, Angew. Chem., Int. Ed., 2009, 48, 344.

29 K. Lang and J. W. Chin, ACS Chem. Biol., 2014, 9, 16.

30 K. Trebesius, K. Panthel, S. Strobel, K. Vogt, G. Faller, T. Kirchner, M. Kist, J. Heesemann and R. Haas, Gut, 2000, 46, 608.

31 E. Demiray-Gurbuz, O. Yilmaz, A. Z. Olivares, C. Gonen, S. Sarioglu, M. Soyturk, S. Tumer, O. Altungoz, I. Simsek and G. I. Perez Perez, J. Pathol.: Clin. Res., 2017, 3, 29.

32 E. Kocsmar, I. Szirtes, Z. Kramer, A. Szijarto, L. Bene, G. M. Buzas, I. Kenessey, P. Bronsert, A. Csanadi, L. Lutz, M. Werner, U. F. Wellner, A. Kiss, Z. Schaff and G. Lotz, Helicobacter, 2017, 22, e12387.

33 J. Joseph, K. N. Baumann, P. Koehler, T. J. Zuehlsdorff, D. J. Cole, J. Weber, S. E. Bohndiek and S. HernandezAinsa, Nanoscale, 2017, 9, 16193.
34 P. Moroz, W. P. Klein, K. Akers, A. Vore, N. Kholmicheva, N. Razgoniaeva, D. Khon, S. A. Díaz, I. L. Medintz and M. Zamkov, J. Phys. Chem. C, 2017, 121, 26226.

35 K. M. Parkhurst and L. J. Parkhurst, Biochemistry, 1995, 34, 285.

36 S. Xu, J. Zhan, B. Man, S. Jiang, W. Yue, S. Gao, C. Guo, H. Liu, Z. Li, J. Wang and Y. Zhou, Nat. Commun., 2017, 8, 14902.

37 G. Knorr, E. Kozma, J. M. Schaart, K. Nemeth, G. Torok and P. Kele, Bioconjugate Chem., 2018, DOI: 10.1021/acs. bioconjchem.8b00061.

38 A. J. Winstead, N. Fleming, K. Hart and D. Toney, Molecules, 2008, 13, 2107.

39 O. Demeter, A. Kormos, C. Koehler, G. Mezo, K. Nemeth, E. Kozma, L. B. Takacs, E. A. Lemke and P. Kele, Bioconjugate Chem., 2017, 28, 1552. 